Portland State University

PDXScholar

\title{
The What and Why of Whole Number Arithmetic: Foundational Ideas from History, Language and Societal Changes
}

\author{
Xu Hu Sun \\ University of Macau \\ Christine Chambris \\ Université de Cergy-Pontoise \\ Judy Sayers \\ Stockholm University \\ Man Keung Siu \\ University of Hong Kong \\ Jason Cooper \\ Weizmann Institute of Science
}

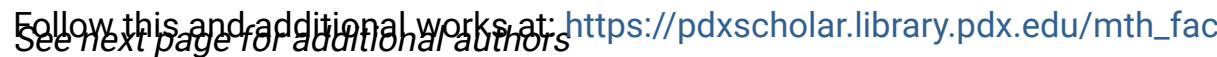

Part of the Science and Mathematics Education Commons

\section{Let us know how access to this document benefits you.}

\section{Citation Details}

Sun X.H. et al. (2018) The What and Why of Whole Number Arithmetic: Foundational Ideas from History, Language and Societal Changes. In: Bartolini Bussi M., Sun X. (eds) Building the Foundation: Whole Numbers in the Primary Grades. New ICMI Study Series. Springer, Cham

This Book Chapter is brought to you for free and open access. It has been accepted for inclusion in Mathematics and Statistics Faculty Publications and Presentations by an authorized administrator of PDXScholar. Please contact us if we can make this document more accessible: pdxscholar@pdx.edu. 


\section{Authors}

Xu Hu Sun, Christine Chambris, Judy Sayers, Man Keung Siu, Jason Cooper, Jean-Luc Dorier, Sarah Inés González de Lora Sued, Eva Thanheiser, Nadia Azrou, Lynn McGarvey, Catherine Houdement, and Lisser Rye Ejersbo 


\title{
Chapter 5 \\ The What and Why of Whole Number Arithmetic: Foundational Ideas from History, Language and Societal Changes
}

\author{
Xu Hua Sun (D, Christine Chambris (D) \\ Judy Sayers, Man Keung Siu, Jason Cooper $\mathbb{D}$, Jean-Luc Dorier (D, \\ Sarah Inés González de Lora Sued (D, Eva Thanheiser (D, Nadia Azrou (D, \\ Lynn McGarvey (D), Catherine Houdement ${ }^{D}$, and Lisser Rye Ejersbo
}

\subsection{Introduction}

Mathematics learning and teaching are deeply embedded in history, language and culture (e.g. Barton 2008). Yet what historical, linguistic and cultural foundations are necessary for the early years of school to adequately prepare children for mathematics learning? To address this question, we summarise work on these three aspects of WNA to frame the entire volume and identify the historical, linguistic and cultural bases on which other aspects of learning, teaching and assessment are based. The chapter provides a meta-level analysis and synthesis of what is known about WNA's foundations of history, language and societal changes, which serves as a useful base from which to gauge any gaps and omissions. This foundation also

The first author was supported by the Research Committee, University of Macau, Macao, China (MYRG2015-00203-FED). The opinions expressed herein are those of the authors.

The first two authors wrote the chapter with the collaboration of other working group members.

X.H. Sun $(\bowtie)$

Faculty of Education, University of Macau, Macao, China

e-mail:xhsun@umac.mo

C. Chambris $(\bowtie)$

Laboratoire de didactique André Revuz, Université de Cergy-Pontoise, Cergy, France

e-mail: christine.chambris@u-cergy.fr

J. Sayers

Stockholm University, Stockholm, Sweden

M.K. Siu

University of Hong Kong, Pokfulam, Hong Kong, China 
provides an opportunity to learn from the practices of different times and languages and from societal changes.

\title{
5.1.1 Conference Presentations: Overview
}

Thirteen papers written by authors from 11 countries were presented for Theme 1 . For presentation and discussion, these papers were divided into four subgroups exploring several overlapping aspects of the why and what of WNA: the historic background of WNA, the language foundations of WNA, the foundational ideas that underlie WNA and the support for societal changes to the teaching and learning of WNA.

\subsubsection{Historical Background}

Zou (2015) summarised findings from historical investigations of arithmetic in ancient China, including how number units were derived and named and how numbers were represented with rod or bead calculation tools and with symbols. Siu (2015) studied the book of Tongwen Suanzhi (同文算指) (Rules of Arithmetic Common to Cultures, 1614) and reviewed how counting rods and the abacus were gradually replaced with written calculations in China. Sun (2015), also discussing early Chinese development, presented the use of advanced number names and

\author{
J. Cooper \\ Weizmann Institute of Science, Rehovot, Israel \\ J.-L. Dorier \\ University of Geneva, Geneva, Switzerland \\ S.I.G. de Lora Sued \\ Pontificia Universidad Católica Madre, Santiago de los Caballeros, República Dominicana \\ E. Thanheiser \\ Portland State University, Portland, OR, USA \\ N. Azrou \\ University Yahia Fares Medea, Medea, Algeria \\ L. McGarvey \\ University of Alberta, Edmonton, AB, Canada \\ C. Houdement \\ Laboratoire de didactique André Revuz, Université de Rouen-Normandie, Rouen, France \\ L.R. Ejersbo \\ Aarhus University, Aarhus, Denmark
}


calculation tools (counting rods and the suàn pán or Chinese abacus) and emphasised how place value is still the most overarching principle of WNA based on Chinese linguistic habit. Traces of this influence can still be found in contemporary core curriculum practices in many countries today.

\subsubsection{Language Foundation of WNA: Regularity, Grammar and Cultural Identity}

Azrou (2015) reported how the historical and linguistic colonisation of Algeria affected the learning of WNA and presented the first step of an intervention for teacher education, which can also promote students' cultural identities. Chambris (2015) showed how changes related to place value that were introduced by the New Math in France (1955-1975) continue to be influential today.

Houdement and Tempier (2015) reported on two experiments for strengthening the decimal (base ten) principle of numeration, assigning a key role to the use of numeration units in France. Changsri (2015) explored first grade students' ideas of addition in two Thai schools in the context of lesson study and an open approach and found that the students used a variety of representations to express addition ideas.

\subsubsection{Foundational Ideas Underlying WNA}

Dorier (2015) gave an overview of the development of numbers, showing how Brousseau's theory can be used in accordance with this historical context to develop the key stages of a teaching sequence using the concept of numbers. Thanheiser (2015), also studying teacher education, adopted the perspective of variation theory and used historical number systems as a tool, finding that prospective teachers developed a more sophisticated concept of the base-ten place value system by examining, comparing and contrasting different aspects of historical systems. Ejersbo and Misfeldt (2015) described research introducing a regular set of number names in primary schools in Denmark. Sayers and Andrews (2015) summarised an eight-dimensional framework called foundational number sense (FoNS) that characterises necessary learning experiences for young children. They demonstrated how to use the framework by analysing learning opportunities in first grade in five European contexts.

\subsubsection{Different Expected Learning and Teaching Goals for WNA}

Cooper (2015) discussed how a university mathematician and a group of elementary school teachers, working together in a professional development course, revealed new insights into division with remainders. McGarvey and McFeetors (2015) 
identified the Canadian public's concerns about the goals of WNA and the support required for students to reach them.

\subsubsection{Working Groups'Discussions}

The eight one-hour sessions were organised in different ways. Examining variation in WNA across history and language and across different communities, working group 1 discussed the implications of different views on the why and what of WNA for instruction and teacher education. Place value in the so-called Hindu-Arabic system was discussed extensively in the working group sessions. In addition to the background discussion and questions posed in the Discussion Document (this volume, Appendix 2), the papers for Theme 1 can facilitate discussions of the following questions:

1. How has the place value concept developed across numeral systems?

2. What are the issues of language and culture in WNA?

3. How did/do different communities change past/current teaching of WNA?

\subsubsection{The Structure of This Chapter}

This chapter describes number representations and their foundational ideas beyond the variations in WNA across history, language and culture. As the world becomes more unified and previously separate cultures interact and begin to merge, incompatibilities become visible and separations arise between different traditions and practices. Many of these incompatibilities can be seen in WNA. Particularly notable is the accommodation needed when traditional language is adapted to deal with the nearly universal decimal place value system for naming and calculating. Approaches to instruction and teacher education are affected by these incompatibilities, as this chapter discusses.

Historical evolution can provide a deeper understanding of the past and present in science as a means of consolidating and clarifying foundations (e.g. Jankvist 2009). We begin with a historical survey of the numeration knowledge development of pre-numeral systems and the conceptual development of numeral systems. We then track the foundational epistemological and pedagogical insights from history. Section 5.2 highlights the differences between cultural practices, especially language, and their links with the universal decimal features of WNA. Post-colonial tensions, where the inconsistency between spoken and written numbers and the incompatibility between numeration and calculation appear, are also explored. Section 5.3 discusses the influence of multiple communities within societies throughout history when attempts at changes are made. Different stakeholder groups in a given society may hold different goals for WNA and thus create different expec- 
tations and support within the society. Various examples are given and key comparisons are made, especially for understanding how and why curricula change.

In parallel with tendencies towards teaching mathematics in globalised ways, a shared awareness has recently evolved among teachers and researchers about the nature of mathematics through the study of its history, traditions and culture. By 'culture', we mean a set of meanings that have been historically constructed, socially transmitted and continually modified and that are embodied in our symbols and language (e.g. Barton 2008). Through this set of meanings, people communicate, perpetuate and develop their knowledge and understanding of life (see also this volume, Chaps. 3 and 9). History and culture shape not only number names and concepts but also the use of numbers in measurement and operations. Different languages have their own syntax and semantics, which emphasise different aspects of numbers; these may foster or hinder a deep understanding of number concepts, especially ideas about base ten, place value and operations. While a purpose of education is to support the continuity of the structures and functions that are unique to a culture and to maintain cultural identity (e.g. Leung et al. 2006), local cultures need to link to universal cultures to avoid isolation in global development. A critical issue, then, is how a cultural system reflects on its own history, language and culture, identifies the disadvantages and advantages of its system, and overcomes its disadvantages and promotes its advantages. What lessons do we learn from these reflections and from the interventions that are based on them?

\subsection{Foundational Ideas that Stem from History}

\subsubsection{Introduction: The Hindu-Arabic Numeral System}

According to some historians, the story of the Hindu-Arabic system (e.g. Lam and Ang 2004) is derived from the Chinese story. See (Chemla 1998) for a different historical perspective and more details in this volume, Chap. 3. This system and its use, which was systematically presented in the Sunzi Suanjing, was transmitted through India during the fifth to ninth centuries, to the Arab Empire in the tenth century and then to Europe in the thirteenth century via the Silk Road (see Guo 2010). Mathematics historians have debated the origins of the Hindu-Arabic numeral system for years. For example, French mathematics historian Georges Ifrah (2000) argued that as the Brahmi notation of the first nine whole numbers was autochthonous and free of any outside influence, the decimal place value system must have originated in India and was the product of Indian civilisation alone. In contrast, Lam and Ang (2004) argued that there is no early Indian text or evidence to show that it was used earlier there than in China. Early texts and evidence show that the Chinese used the rod numeral system continuously for almost 2000 years. This historical fact is not well known in either the Western or Eastern communities of math education because of the limited dissemination of the conceptualisation history of place value of the Hindu-Arabic system. 
According to Lam and Ang (2004), in Western Europe, before the advent of the Hindu-Arabic numeral system, few mathematicians would have been able to perform multiplication. In contrast, in ancient China, the operation of multiplication would have been commonly known as far back as the Warring States period (475221 BCE), not only among mathematicians but also among officials, astronomers, traders and others. This could be because the Chinese rod number system used the concept of place value. It is not surprising that the Nine Chapters on the Mathematical Art includes common fractions, areas, the rule of three, least common multiple, extraction of square and cube roots, volumes, proportion and inverse proportion, relative distance and relative speed, surplus and deficit, rule of false position, the matrix notation, negative numbers, simultaneous linear equations and right-angle triangles because it was grounded in the advanced decimal place value system (Chemla, 2007).

A culture's arithmetic development may be confined or promoted by the numeral system used. For example, multiplication with large numbers could not be well supported by a simple tally system. The Hindu-Arabic numeral system is much more complex than others: it includes a principle for naming numbers, which is ten based with multiple units, and the additive and multiplicative relationships are embedded implicitly, with only the digits recorded. It is universally used in the world because every number, however large, can be easily represented and computations can be easily realised.

According to the Discussion Document (this volume, Appendix 2), historical reconstruction was in the foreground of WNA. For a better understanding of this system, a brief conceptual development of the numeral system ${ }^{1}$ is examined, and associated epistemological and pedagogical analyses are carried out below. This study has two motivations: to understand the foundations of established whole number arithmetic (product) by studying the historical origins (process) and to provide insights for modern teaching by investigating epistemological obstacles (this volume Chap. 9, esp. Sect. 9.3.2). Jankvist (2009) argued that 'history can not only help to identify these obstacles, it can also help to overcome them: an epistemological reflection on the development of ideas in the history can enrich didactical analysis by providing essential clues which may specify the nature of the knowledge to be taught, and explore different ways of access to that knowledge' (p. 237).

We examine the foundational idea of number representation development and do not describe all of the historical facts. The conceptual development of numeral systems can be classified into four types based on conceptual development progress: the tally system, additive system, multiplicative-additive system and decimal place value system. In each case, different strategies to realise operations were invented; these are explained below. Progress could better mirror the development of number structuring and some epistemological obstacles in history/learning (e.g. Jankvist, 2009).

\footnotetext{
${ }^{1}$ A numeral system (or system of numeration) is a writing system for expressing numbers - that is, mathematical notation for representing numbers of a given set using digits or other symbols in a consistent manner.
} 


\subsubsection{Knowledge of Pre-numeral Systems}

\subsubsection{Early Numeration Practices}

Many anthropologists (e.g. Ifrah 2000; Menninger 1969) have found that some ancient cultures did not develop numbers at all. Some had names only for one and two and some up to three or four. Larger numbers were described as 'many'. In many ancient languages, words for 'two' or 'three' exist between singular and plural as a means to distinguish one from many, which is the beginning stage of the development of numeration.

One-to-one correspondence between an organised list of words - that is, the list of number names - and the units of a collection is typically considered an elementary process in counting, and it is the most fundamental stage. In many cultures (e.g. Menninger 1969), parts of the human body have been used to make one-to-one correspondence, often starting with fingers. Despite having limited sets of number names, some cultures developed quantitative practices that go beyond the greatest number available, such as the use of tally systems, and partitioned large quantities into smaller countable quantities (Baxter 1989). A society's early numerical practices are embedded in its development of a tally system, which is built on ordinal numbers, cardinal numbers and the counting principle - namely, one-to-one correspondence (Seidenberg 1962). Beyond tally numeral systems, various cultures developed different numeration systems, yet all had in common a symbol for one, the unit of ones and other symbols for collections of that unit (May 1973).

\subsubsection{The Invention of the Counting Principle}

Knowing whether quantities have increased or decreased was likely a key problem for many ancient tribal peoples. To recognise more or fewer, one of the earliest methods directly stimulated the invention of one-to-one correspondence with an intermediate collection of stones (Dorier 2015). Before representing and naming numbers, people developed several ways to evaluate quantities beyond rough estimation. For example, we can imagine that shepherds were concerned about the possibility of losing sheep when they returned from the fields at night. We can only speculate how the use of stones became tokens in one-to-one correspondence, but there is clear evidence that tokens were used in one-to-one correspondence by the use of 'envelopes holding counters to represent sets' of numbers (SchmandtBesserat 1992, p. 190). Illustrations of artefacts were used to form records of number of tokens as representations. Several other artefacts, some dating to Palaeolithic times $(15,000 \mathrm{BCE})$, such as notches and bones, are indicators of human activity related to the building of corresponding collections with specific cardinal numbers to record quantities. This may have been the beginning of the invention of the counting principle. 


\subsubsection{The Pre-structures of Number Naming}

Because of rhythmic demands of oral pronunciation, no language represents numbers by articulating the same sound more than two times. That is, there are no known examples such as 'one one one one' for four or 'three three three' for nine. Words are sometimes repeated but not more than once (Cauty 1984; Guitel 1975). This implies that number names are not based on the principles of a tally system (a numeration system of keeping a record of quantities and amounts by using single strokes to represent the objects being counted) (see Sect. 5.2.2 and this volume Chaps. 9 and 10). If number names exist in a given language, the list of the names of the smallest numbers is a sequence of words that is more or less long, and the words are more or less independent. Conversely, if a given language presents number names for large numbers, sequences of number names beyond a threshold always have a multiplicative structure. The threshold is almost always under 100 (Cauty 1984; Crump 1990; Menninger 1969). In Chinese, and other languages such as Chunka (González and Caraballo 2015), the threshold is ten.

We consider the sequence before the threshold, as its structure is of interest. Cauty (1984) identified several types of sequences within spoken numbers:

1. Ordinal - a list of words independent of each other.

2. Ordinal with benchmarks - as described above, it begins with a list of words and is followed by benchmarks on a scale, e.g. the Panare language has benchmarks for 5, 10, 15 and 20 and counts from 1 to 4 between the benchmarks (Cauty 1984).

3. Cardinal with addition - where a number is represented by juxtaposed number names whose sum is the given number (such as how XXIII means two 10s and three 1s in Roman numerals).

4. Cardinal with multiplication - where a number is represented as a sum of products of small numbers ('digits') times units (such as how three hundred two means three times one hundred and two in Chinese spoken and written numerals).

The Oksapmin people (Saxe 1981) use body parts to recognise numbers, moving from the right-hand fingers up to the right eye - the first finger is 1 and the eye is 13 . The nose is 14 , and they move symmetrically from the left eye to the left-hand fingers to count from 15 to 27. This can be considered a long ordinal list. According to Cauty (1984), the ordinal-with-benchmarks type above is often confused with the cardinal type with addition/multiplication. The difference between the two lies in the grammar, which indicates movement in relation to the benchmarks. Benchmark and additive numeration may be the beginning of the idea of a base (as in base ten). However, in some languages, the names of larger numbers may be expressed in terms of smaller numbers and arithmetic operations, which may be the beginning of the exploration of number structure, e.g. $3=2+1$ and $5=2+2+1$, and even multiplicative forms such as $6=2 \times 3$ and $18=3 \times 6$. However, it is rare for 2 to be expressed as $1+1$ (Crump 1990). In Nigeria, the Yoruba numeral system is based on 20 , and other numbers may be expressed by subtraction, e.g. $35=(20 \times 2)-5$. 
This can also be seen in Roman numerals, where, for example, CX means 110, but XC means 90.

To meet the need for counting, various cultures developed the counting principle and one-to-one correspondence and named numbers with some regularity, sometimes using addition and/or multiplication, to specify quantity. From these practices, we can learn about the conceptual development of number systems, as naming and operations (addition/multiplication) were developed at the same time. The counting principle, invented number names and written number symbols are needed for the development of a formal numeral system. However, the coordination between cardinal numbers and ordinal numbers could be considered too trivial, too easy to explicitly design in many curricula and lessons. These could be reasons for longterm learning difficulty in later number development and operations.

\subsubsection{The Conceptual Development of Numeral Systems}

\subsubsection{Tally Systems}

The Ishango bone ${ }^{2}$ notches (about 18,000 to 20,000 BCE), ancient Chinese knots in string and Sumerian marks on a clay tablet show what appear to document quantity as an 'early stone or agricultural age' vision of numerals (Mainzer 1983/1991) in almost all ancient cultures (see also Sect. 9.2.2). Ifrah (2000, p. 64) points out that tally systems could be an early vision of systematic counting numbers 'first used at least forty thousand years ago'. These could be the origins of Roman or Etruscan numerals (Ifrah 2000, pp. 191-197). Such marks clearly point to the development of ancient written number representations. Tally numeral systems are among the most primitive means of recording quantity (Hodgson and Lajoie 2015). Counting up and down might be the natural calculation of sums and differences. It is the simplest (unary) numeral system, and it plays an important role in the fundamental counting action of building one-to-one correspondence between objects and names, forming a set of reciting numbers in ascending order. This could be how the first systematic conceptualisation of numbers in a set of numerals developed. Tally systems directly reflect the fundamental idea of counting for small numbers: one-to-one correspondence. Once tallying becomes an established practice, establishing a set of standard names for small numbers might be the next step, as it affords the ideas of both cardinal numbers and ordinal numbers and allows the description of a collection of objects arranged in a particular order. The analysis above indicates that counting principles could be key to the conceptual development of numeral systems. Their absence could result in counting by rote memorisation, skipping objects, counting randomly and counting an object twice or multiple times.

\footnotetext{
${ }^{2}$ The arithmetic interpretation of the Ishango bone has been recently contrasted by Keller (2016), claiming that further studies and references to archaeological finds would be needed.
} 


\subsubsection{Additive Systems}

As counting needs for large numbers increased, the difficulty of remembering many number names led people in many societies to the idea of grouping ones, using special abbreviations for repetitions of symbols and inventing a new object or symbol to signify this quantity (Groza 1968). This grouping may be the first step in coherently combining the different records to make structural organisation common across counters (Bass 2015). It denotes the mathematical abstraction of the numeration system in which the number represented by written numerals is simply the sum of the value each numeral represents. It also requires new symbols for different groups of ones and new strategies to enumerate the new collections. The different sized groups could be the beginning of the idea of multiple units. This kind of grouping - or re-counting different cardinal collections - was developed in all ancient civilisations to count large numbers (e.g. Bass 2015). Sumerians ( 3500 BCE) initially used a tally system comprised of collections of small cones to represent collections of items (Schmandt-Besserat 1992). Over time, they replaced 10 small cones with a small ball, 6 small balls (or 60 small cones) with a larger cone and 10 larger cones with a cone of the same size with a round hole in its centre, thus using a mix of bases 10 and 60 . These objects were packed in a spherical clay container that had to be broken to identify the inside number. Later, these objects were represented with marks on the surface. Eventually, the objects were abandoned and only their representations were used. Using cuneiform features, marks of wedges and corners - specifically, one vertical wedge for 1 and one corner for 10 - were written on a clay tablet. Dating to around $3300 \mathrm{BCE}$, this could be the first known written additive numeral system. An updated numeration system was constructed with a set of symbols, called numerals, together with a set of rules for writing to represent numbers.

Around 1500 BCE, the Egyptians invented a hieroglyphic written additive numerical system in base ten. Around the fourteenth century BCE, the main concept used in most Chinese numerals in the oracle bone script found on tortoise shells and animal bones was grouping, which partly formed an additive system. It is interesting to note the numerals for 2 and 3 , are still used in daily language. Ancient Roman numerals, such as CXXXV for 1 hundred, 3 tens and five, recorded numbers using the concept of grouping ones and regrouping into higher units. Although they used different grouping approaches at each step, many cultures (Sumerian, Babylonian, Egyptian, Greek, Roman, Arabic, Chinese, Mayan, Aztec, etc.) developed or used many-levelled additive numeral systems based on the principle of successive grouping. Thus, historically, many numeral systems were developed by progressing from tally systems to additive (grouping) systems in which multi-units with additive relations (no multiplication relations) were developed. The analysis above indicates the understanding of multi-units as necessary to deal with large numbers and of the possibility of learning difficulty in counting because of the different units and unit conversions from the tally system. 

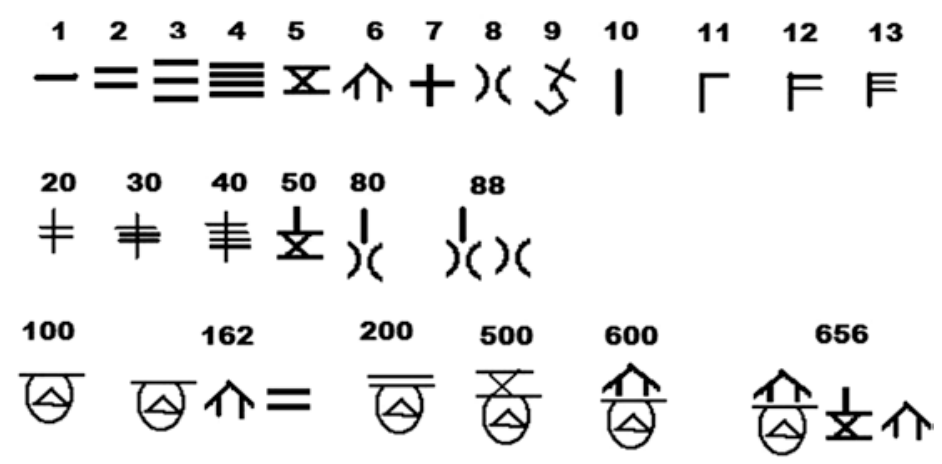

\section{$\begin{array}{llll}1000 & 2000 & 3000 & 4000\end{array}$}

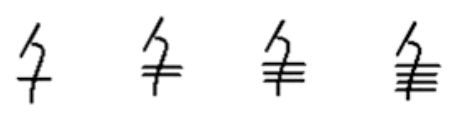

Fig. 5.1 Shang oracle bone numerals from the fourteenth century BCE

\subsubsection{Multiplicative-Additive System}

The additive systems above have been rather common inventions. Many historical examples indicate that the multiplicative concept, most often with irregular forms at the beginning, has also been a common invention for representing larger numbers with a simpler approach. Counting the signs in the additive form and then naming the value of the sign lead roughly to a multiplicative-additive system. For example, the Roman numeral CCC (300) is called trecenti in Latin, from tres (three) and centum (hundred). It is a numeration system in which the number value should be the sum of the products of units indicating how many of each unit are considered, where the multiplicative notion is added. Here, $\mathrm{C}$ is a unit rather than a number in an additive system. In such systems, there is a different symbol for each power of ten and for each number from one to the base minus one.

The ancient Chinese numeral system found on bones and tortoise shells of the late Shang dynasty in the fourteenth century BCE was the first multiplicative-additive system based on the decimal system and was both additive and multiplicative in nature. Here is a selection of Shang oracle bone numerals (Martzloff 1997; Needham 1959) (Fig. 5.1).

Here, 200 is represented by the symbol for 2 and the symbol for 100, 3000 is represented by the symbol for 3 and the symbol for 1000, etc. (Fig. 5.2). The additive nature of the system means that symbols were juxtaposed to indicate addition, so 4359 was represented by the symbol for 4000 followed by the symbol for 300 , the symbol for 50 and the symbol for 9 (Fig. 5.3).

As this was not a positional system, there was no need for a zero (Fig. 5.4).

Guitel (1975) classified this as a hybrid system. A number of additive systems evolved into multiplicative-additive systems (Chinese, Mayan, etc.), but most 
Fig. 5.2 Representation of 1234 on a horizontal counting board

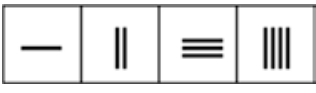

Fig. 5.3 Representation of 4359

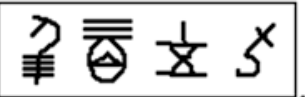

Fig. 5.4 Representation of 5080

remained additive (e.g. Roman written numerals, Egyptian, Greek). Multiplicative systems improve calculation speed. However, the difference between multiplication and addition could cause concept confusion and learning difficulty, and number naming using both multiplication and addition could present an epistemological obstacle.

\subsubsection{Decimal Place Value System}

Both the decimal numeral system and positional notation or place value notation can further simplify arithmetic operations because of the use of the same symbol for different orders of magnitude (e.g. the 'ones place', 'tens place', 'hundreds place'). By using 1, 10 and 100 as numeration units (not number names in an additive numeral system) and both multiplicative and additive concepts, a much more advanced numeral system, the decimal place value system, was invented, in which a number can represent quantity with multiple decimal units. Both the digit itself and its referring numeration unit determine the value that a digit represents. The numeration unit that a symbol occupies determines the value of the unit, and the symbol itself determines how many of these units are being represented (Groza 1968). Using numeration units, calculations in the place value system are quite different from those in the tally and additive numeral systems. The numeration units and their conversions are the key to calculations. Addition should be carried out with two numbers with the same units, and numbers with different units should be converted to the same units using the following conversion rate: 1 thousand $=10$ hundreds; 1 hundred $=10$ tens ( 1 in the third place); 1 ten $=10$ ones $(1$ in the second place); etc.

The Chinese counting rod system and the Hindu-Arabic numeral system are decimal place value systems (Japan, Korea and Thailand imported the Chinese decimal system (Lam and Ang 2004)). About the fourth century BCE (the West Zhou dynasty), the first place value system using counting rods came into use (Guo 2010; Martzloff 1997). Numbers were represented by small rods made from bamboo (Zou 2015) and used on a counting board (this volume, Chap. 3). A number was formed in a row with the units placed in the rightmost column, the tens in the next column to the left, the hundreds in the next column to the left, etc. (Fig. 5.4). 
A zero on the counting board was simply a blank square. Sun Zi's Suanjing (孫 子算經 $500 \mathrm{CE}$ ), the earliest extant treatise, described how to perform arithmetic operations on the counting board and gives instructions on using counting rods to multiply, divide and compute square roots. Though humans have always understood the concept of nothing or having nothing, the symbol of zero was used to represent the 'zero' first in the Hindu-Arabic numeral system. This was the first time in the world that zero was recognised as a number of its own, as both an idea and a symbol (Martzloff 1997). That place-value notation with the same ideas of zero emerging in two very different settings aimed to make more efficient systems to represent any number. Xiahou Yang's Suanjing (夏侯陽算經500 CE) explains not only positive powers of 10, but also decimal fractions as negative powers of 10 (Martzloff 1997), applying positional notation to the decimal fraction ring. Thus, decimal place-value notation emerged as a more efficient system for calculations of both whole numbers and fractions.

\subsubsection{Modern Theoretical Approaches}

Below, two schemes or 'theories' of place value numeral systems are proposed to describe written positional systems. The first is classical theory. It belongs to traditional arithmetic treatises, such as those of Bezout and Reynaud (1821) and Ryan (1827). This theory has been used in France to teach positional notation for centuries. The second theory belongs to academic mathematics.

The words for units used in numeration - that is, the words 'ones', 'tens', 'hundreds' and so on - are henceforth called numeration units. Numeration units are built one after the other in the following way. (1) The first ten numbers are built one after another, starting with the unit one and then adding one to the previous number, forming the numbers one, two, etc. (2) The set of ten ones forms a new order of units: the ten. (3) The tens are numbered like the ones were numbered before, from one ten to ten tens: one ten, two tens, etc. (4) Then the first nine numbers are added to the nine first tens: one ten, one ten and one one, one ten and two ones, ... two tens, two tens and one one, and so on, forming the first 99 numbers. (5) The set of ten tens forms a new order of units: the hundred and so on. Numbers' names are presented as a literal translation built on units' names (adapted from French): 'Say the tens, then the ones'. For example, as three tens is thirty and four ones is four, then three tens and four ones is thirty-four. The rules have exceptions, however. For instance, the usual name of ten-one is eleven. Finally, after building the numbers, the positional notation is stated. To write numbers without writing the units' names, it is sufficient to juxtapose the numbers of units of each order with the ones on the right side; then, each place represents a unit that is ten times larger than the one on its right. Places that are not represented are marked with the sign 0.

The current reference knowledge for place value in academic mathematics is based on the polynomial decomposition of a whole number $n$ in a given base $r$ : $n=\sum a_{i} r^{i}, 0 \leq a_{i}<r$, which is a much more generalised expression of classic theory with the particular formal abstractions that characterise modern mathematics. The 
current proof of the existence and uniqueness of the decomposition in a formal way involves Euclidian division. The positional notation is defined as the juxtaposition of the coefficients of the polynomial. This theory, which belongs to advanced algebra, is henceforth called academic theory (following Bezout and Reynaud 1821).

Both of the approaches presented above provide multiplicative descriptions of positional notation. The multiplicative description and the recourse to exponents are not necessary when devices for computing, such as the suàn pán or abacus, are used (see this volume, Sect. 9.2.2), as the device itself embodies this convention.

In sum, the decimal place value system and computation were handed down to us by our ancestors and underwent improvements over time. It is worth determining which aspects were improved and the reasons for the changes. We have described several steps, each requiring concept and relation development. From the tally system to the additive system, multiunit notions - that is, the grouping of units - are critical. Grouping units simply means a counting process using larger units, which must also rebuild the multiplicative relation with lower units. Multiplication also further simplifies the repeated addition relation, which advances the abstraction process of counting. The development of this elegant positional base-ten system took place over a long time; therefore, the fact that understanding numbers is complicated should not be surprising. Without this notation, one would encounter the same difficulties that peoples of ancient cultures encountered in large number and fraction development.

\subsubsection{Epistemological and Pedagogical Insights from History}

\subsubsection{Pedagogical Insights from the Pre-history of Numbers}

The pre-history of numbers and the invention of small numbers can provide insights into the beginning of the teaching of numbers and learning by young children. Oneto-one correspondence is likely an essential step towards the concept of numbers: the recognition of quantity as a property of collections. History as described in the previous section(s) shows a double role of one-to-one correspondence: the intermediate collection of objects such as stones and the intermediate collection of words, e.g. the number names. As Dorier (2000) advised, history can be used to reconstruct an epistemologically controlled genesis that takes into account the specific constraints of the teaching content. The fundamental situation of numbers conceived by El Bouazzaoui (1982) and Brousseau (1997) belongs to such a programme. It has to do with quantity (not yet numbers) and can be expressed as building the same cardinal number collection to a given collection. Typically, the task is, 'Look, there are rabbits here. Go and bring carrots so that each rabbit can eat. That is: each rabbit should have one carrot, no more, no less'. It can be observed that spontaneously, a young child does not count even if she knows a sequence of number names. From this general situation, several steps can be conceived, taking into account didactical variables (Brousseau 1997) - that is, conditions on the tasks to be achieved that can 
change what children learn. For example, are paper and pencil available? If so, children can draw carrots, thus making a list of what is needed, which is an intermediate collection. How is the size of the collection - e.g. [2-6], [6-12] and [12-100] related to children's knowledge of the sequence of number names? Are the rabbits visible from where the carrots are? They can be drawn and arranged in specific ways, such as on a die or in several areas of a sheet of paper to foster concepts of, for example, three in one area, two in another and so on (Briand et al. 2004; Margolinas and Wozniak 2012).

\subsubsection{Understanding Numerals' Uses: To Write, to Compute, to Talk}

Not all systems have followed the same development. For example, China never had a recorded additive system. It is interesting to note that in the West, written Roman numerals are additive, while the corresponding spoken number names are multiplicative. For example, XXX is trĭginta in Latin, where tres is 3 and decem is $\mathrm{X}$ (ten). Spoken numerals, especially for large numbers, belong to a pre-multiplicative-additive system. However, in contrast to speaking and writing, the number concept for computation is positional.

The positional principle for the Old Babylonians (ca. 2000-1600 BCE) was in base 60 . Its digits are of the written additive system in base ten as they are all under 60. In the scribal school of south Mesopotamia (ca. 2000-1800 BCE), positional notation was only used for computation, never to express measurements, which used only rather small numbers, written in additive form, associated with a developed system of units (Proust 2008, 2009). The ancient Romans and medieval Europeans did not write positional numbers but used the additive system of Roman numerals even though they computed with a positional abacus, which embodies the positional principle used by the Greeks, Old Babylonians (Høyrup 2002) and Chinese (Fernandes 2015). The position of each digit within a number denotes the multiplier (power of 10) multiplied by that digit. These tools were constructed using principles similar to the abacus: a board with columns into which identical objects are put, where all of the objects in a given column indicate the same value, which is generally that of a digit of the additive system (or of the unit if it is a numeration unit system), where adjacent columns contain objects representing two successive digits (two successive units in a numeration unit system). A key feature is that if the ratio of the digits between two adjacent columns (with the lesser on the right) is $n, n$ objects in the right column can be replaced by one object in the adjacent left column without changing the number. If the ratio between the columns is $n$ (which would thus be the base of the system), one simply moves the objects in the adjacent left column and multiplies by $n$.

The case of the Incas features incompatibilities between written numbers and calculations. The Mayans and Aztecs developed a base-20 numerical system, while most of the cultures in the Andean region developed a base-ten system. The Incan civilisation used a cord system to make alphanumeric records to code information and solve numerical problems. These quipus are systems of strings with different 
colours and knots. The position of the cords, the types of knots and the colours of the strings are elements of its logical-numerical nature (Ascher and Ascher 1981). However, the Incas used the yupana to make calculations using a base-ten system (González and Caraballo 2015).

When using a counting board, the successive steps of a computation in progress disappear. In the Old Babylonian case (ca. 2000-1600 BCE), as in the Chinese one, it seems that the positional notation was not used to record quantities. In the Old Babylonian tablets of Nippur (school of scribes), positional notation writing seems to have been used to indicate computational algorithms (Proust 2009) or to record the steps of a computation on a tool (Høyrup 2002; Proust 2008). In ancient Chinese books, writing with positional notation was used to explain the locations of the rods or beads on the tool and especially to explain computational algorithms (Chemla 1996; Lam and Ang 2004).

From the survey above, we come to the surprising conclusion that in many Western civilisations, to express large numbers, the written systems are additive, and the spoken systems are multiplicative, while the tools used for computations embody a positional principle. In other words, a large number written in an 'additive' way is often 'spoken' in a multiplicative way and, if used in a computation, is expressed in a 'positional' way using physical structure rather than written symbols. The invention and subsequent widespread adoption of the Hindu-Arabic system changed this, as this unique system is used to write and compute both positionally and multiplicative additively.

\subsubsection{Understanding the Conceptual Changes in the Development of the Decimal Place Value System}

Memorising the Multiplication Table

Ancient Egypt, ancient India and ancient Rome did not develop multiplication tables because of the additive systems they used. However, ancient China, Greece and Old Babylon did (Høyrup 2002; Menninger 1969). It is reasonable to imagine that the multiplication concept could be difficult in the current Western curriculum (Beckmann et al. 2015). In 1989, the National Council of Teachers of Mathematics (NCTM) developed new standards recommending reduced emphasis on the teaching of traditional methods relying on the rote memorisation of multiplication tables. NCTM made it clear that basic multiplication facts must be learned in the Common Core State Standards for Mathematics in the USA (Common Core State Standards Initiative, 2012). Historically, in China, the multiplication table has been a more stable part of the base-ten rod and abacus calculation curriculum, and there has been no controversy about memorising it (Cao et al. 2015). It is regarded as the foundation of multiplication/division column calculation and plays an important role in calculating rapidly and accurately (Cao et al. 2015). It includes the essential facts of column multiplication, which are similar to the function of number naming 1-9 for addition and subtraction. Therefore, the importance of memorising 
the multiplication table for multiplication/division calculation curriculum development and instruction should not be underestimated.

\section{Unit Conversions}

According to the above analysis, the one-to-one relationship of adjacent unit types in the additive system needs to change to the ten-to-one relationship of the place value system. In a simple additive system, each symbol has a fixed face value. Because in the base-ten place value system, the value that a digit represents is determined by a combination of two factors, its own face value and its reference unit within a numeral, a single symbol can represent different values depending on its referent unit. By this use of numeration units, calculations in a place value system are quite different from those in tally and additive numeral systems. The numeration units and their conversions are the key to calculations: addition should be carried out with two numbers with the same units. Numbers with different units need to be converted into the same units by the conversion rate 1 thousand $=10$ hundreds, 1 hundred $=10$ tens ( 1 in the third place), 1 ten $=10$ ones ( 1 in the second place). Therefore, the composition and decomposition of a higher unit is a key procedure for addition/subtraction that cannot be realised by the counting approach in tally and additive numeral systems. Thanheiser (2015) designed an intervention based on a history of numeration with successive steps - tally, additive and positional baseten systems - and then performing computations with these systems to reinforce place value for teacher education. Thanheiser argued that the unit conversions can be difficult because they do not develop naturally in calculations in the additive system: counting on, counting down and the doubles approach.

\subsection{Foundational Ideas from Language and Culture}

Number language is the first cultural symbol a human encounters in mathematics, and it appears more salient than other teaching and learning factors in relation to WNA. The notions of numbers are first learned as a linguistic routine of counting through which the number names are perceived as sign systems or cultural semiotic systems that enable the symbolic representation of knowledge (Goswami 2008). There are few studies on conflicts among number naming, cultural identities and universal decimal features and how local languages are linked to universal HinduArabic numerals. In the following, these two questions are discussed. 


\subsubsection{Whole Number Naming: Universal vs Cultural}

A clear example of such conflict is in whole number naming, where history and culture shape not only number names and conceptualisations but also their use in measurement and operations. When analysing this phenomenon, two situations emerge: the first one is what remains from ancient times, which contaminates a more recent and coherently organised cultural system at the oral level, such as the case of spoken numbers in Denmark. The second situation relates to the lively colonial process related to different uses of language fed by political and social interactions that continuously develop over the years, such as spoken and written numerals in post-colonial areas such as Algeria and Guatemala. Analysis of the two situations is possible thanks to direct reports in the working group and provides interesting insights into the historical evolution of elementary arithmetic and how it can affect number names and result in learning difficulties. Importantly, both situations may offer opportunities to enhance the learning and teaching of numbers and contribute to the awareness of a cultural identity if sensitively handled by teachers.

\subsubsection{The Danish Case: The History of Number Names in Denmark}

As presented by Ejersbo and Misfeldt (2015), in a typical situation, the effects of the past on spoken number names in Denmark have led to problems for many children. Some number labels are rooted in ancient names, reflecting a primitive non-decimal system. One hundred years ago, 'eighty' was said as 'four times twenty', but in daily speech, it became 'firs', which is close to 'fire' or 'four' in Danish. About 50 years ago, this abbreviation was made official, as 'times twenty' was dropped for counting numbers, although it was retained for ordinal numbers. 'Seventy' in Danish is said as 'half four' and previously as 'half four times twenty'. 'Half four' is actually a half taken to four (from three), so it means 'three and a half' (as in German time counting, where 'half four' means 'half past three'). Thus, as Danish practices inversion for spoken numbers between 13 and 99, 'seventy-three' is said literally as 'three and half-four'. As the old roots are unknown to most students, the names of the tens up to 100 are taught through rote learning, and the underlying rules are not addressed. This has caused difficulties for Danish students, many of whom are insecure about reading and writing two-digit numbers for their first 3 or 4 years of schooling (Ejersbo and Misfeldt 2015).

Thirty years ago, it was common practice to address these difficulties at a cultural level by offering opportunities both for teachers and for children to reflect on the roots of the mathematical notion. Examples were used at cognitive and pedagogical levels to highlight relevant features, such as the present decimal positional system (base 10 in comparison with base 20) and the additive nature of representation in a given base. Today, these opportunities are not exploited in the first years of school, as there was little evidence to support the learning of the number names (possibly due to how the teaching had been organised). Danish students continue to 
struggle with combining the words for a number, its cardinal value and its digit representation. Thus, while, a language changes according to how it is spoken in everyday life, it seems difficult to change number names because of cultural continuums, even though doing so would help students better understand numbers.

\subsubsection{The Algerian Case: Language Diversity in the Post-colonial Era}

Many previously colonised countries retain the influences of their old political masters, which are often reflected in their educational systems, such as in the organisation of schools, the content of the curriculum and the languages in which specific subjects are taught. Young children learn to count in their native language, and this is encouraged until they begin formal schooling. Thus, in their early years, when teachers seek to develop a strong number sense, they can find it challenging to learn WNA in a 'foreign' language. Unfamiliar words and new conceptualisations often lead to identity problems for children.

Azrou (2015) studied the situation in Algeria. Over the last 50 years, as the power and cultural relationship between France and Algeria changed, a range of decisions were made by the authorities regarding the teaching of mathematics at different levels. For example, at the university level, all mathematics is taught in French, but at the school level, while formulae are written in the Latin alphabet and read left to right, mathematical terms are written and spoken in Arabic (right to left). The difficulties young children experience are exemplified in the Algerian context. Four languages are spoken (classical Arabic, dialect, Berber and French), and oral and written words are shared differently by different social groups. For example, although numbers are taught in classical Arabic in schools, some children from particular communities speak only a dialect and/or Berber. At this stage of schooling, because of inversion, children are expected to write numbers from the lowestvalue digit to the highest - that is, from right to left like word writing. This expectation changes at grade three, at which point all children are expected to engage with numbers not only in Arabic but also in French, where words are written from left to right and where there is no inversion above 16. Some children write numbers from the lowest to the highest-value digits in the direction of word writing for both languages.

The analysis of these two situations provides some insight into the language evolution of elementary arithmetic and how it can affect number naming and result in learning difficulties. Obviously, whole number language conflicts between universal, cultural and colonial naming can cause meta-level learning difficulty. Therefore, a bridge between curriculum and instruction is needed to link local languages to universal Hindu-Arabic numerals. 


\subsubsection{The Incompatibilities Between Spoken Numbers, Written Numbers and Numeration Units Within 100}

When Anglophone speakers learn how to tell quantities and then write (in digits) 'what they hear', they are faced with the difficulties of two changes in the number structure: 11 and 12 sit outside the 'teen' numbers, 13-19, where an inversion structure in the spoken names (the ones-place digit, not the tens-place digit, is spoken first) is used (thirteen, fourteen, fifteen, sixteen, seventeen, eighteen and nineteen). There seems to be no logic to this disturbance to what is otherwise a strong pattern in English's number system in English. Thus, these words have to be learned and remembered. In contrast, the Chinese system provides a logical way of reading: 11, 12 and the 'teen' numbers (ten-one, ten-two, ten-three, ten-four, ten-five, ten-six, ten-seven, etc.) do not need learning or remembering in quite the same way. Another difficulty is the inversion structure (as demonstrated in the Algerian and Danish cases above) between spoken numbers (e.g. the ones are said before the tens in 14) and written numbers (e.g. the tens are written before the ones in 14), which could cause learning difficulties. The cognitive challenges of the number learning of 11-19 have long been recognised as 'the trouble with teens' (Miller and Zhu 1991). This might relate to the controversial question of place value or quantity value in the Western curriculum (e.g. at what stage should the 3 in 38 be identified as 3 tens rather than 30 (Askew and Brown 2001)?).

Houdement and Tempier (2015) identified three number systems in the process of knowing numbers: written numbers (written as 56, showing face value), spoken numbers (spoken as fifty-six, showing quantity value, number size made of ones) and numeration unit numbers (presented as 5 tens and 6 ones, showing place value, number size made of numeration units). Generally, a spoken number system is rooted in local oral language, and it naturally follows the grammar of local language and thus directly shows cultural identity. It is often irregular in Western languages (72 = soixante-douze in French, i.e. sixty-twelve). It is mainly learned at home as an inherited mother language. The spoken names are developed as sounds connected to the numbers of objects in the sets. Written numbers are mainly learned at school as a second language. Teaching the third system (the numeration unit numbers, showing place value) specifically and gradually in relation to the two others might facilitate understanding of (1) the base-ten place value system (Tempier 2013), (2) computation algorithms (Ma 1999) and (3) the decimal form of rational numbers. In English, numbers' names are those of the units, but not in French. In France, the names of the second, third and fourth decimal numeration units for whole numbers are not used in the everyday language (Chambris 2015).

In many languages of East Asia and elsewhere throughout the world (such as several African languages), spoken numbers are similar to numeration unit numbers. Written numbers are numeration unit numbers without the numeration units, so the relationships among them are simpler than the European ones. In the HinduEuropean language area, students must memorise many spoken, written and numeration unit names without connecting them logically with the written names ('ten' is 
not heard between 11 and 16). A way to achieve a logical link is to translate written names into numeration unit names and vice versa. This is related to the role of numeration units in the curriculum in many countries. For example, in America, Fuson, Smith and Lo Cicero (1997) explored 'tens' and 'ones' in English (and 'decenas' and 'unidades' in Spanish), but did not refer to 'units' but to 'tens-andones words' and also to 'position' in Spanish. In French textbooks, numeration unit names - e.g. ones, tens and hundreds - disappeared from the curriculum in the 1970s; they may remain as place names. It is frequent not to find the relation 1 hundred $=10$ tens in current French second and third grade textbooks (Chambris 2015). Tempier (2013) observed three third grade teachers and found that only one of them explicitly referred to the relations among units, although all three used the units' names to describe places. Numeration units completely disappeared as units in the 1980s, but they remained as places (position) and may have reappeared in other forms and practices since 1995.

\subsubsection{Links and Incompatibilities Between Numeration and Calculation}

It seems that hundreds and thousands play a more visible role in spoken Western languages than ones and tens because the latter do not function as units in many spoken languages within 100. Thus, an opportunity to build an understanding of the numeration structure is lost. As mentioned above, the Chinese language stresses that spoken numeration recalls both the number name and numeration unit based on the language categories of classifiers (Sun 2015), which specifies the value of digits in a clearer way. The corresponding teaching and learning of numbers and calculations emphasises how to compose/decompose a numeration unit by using a composition or decomposition approach (e.g. 1 ten $=10$ ones). Compared with many Western curricula, counting and memorising number facts is emphasised, especially in the teaching and learning of 1-2 digit calculations. In Chinese, the result of $8+7$ is said as ten-five. The number name fosters the use of the make-a-ten method: eight plus two, ten plus five as the result is given in the procedure. In the same manner, to compute $40+10$, one has to say that four tens and (one) ten is five tens. If the language does not stress the unit of tens, children will obtain various answers. For example, in French, $8+7$ may be $7+7$ (double) fourteen and one. Young children memorise 'doubles' rather quickly, and then there is only one (easy) step left to find 15. To make a ten to compute $8+7$ requires the following: (1) to make ten with 8 (take 2), (2) take 2 from 7 (5 left) and (3) transform 10 and 5 into 15. This last step does not exist in Chinese, but it does exist in languages that do not use ten to compose the names of numbers between 11 and 19. In many European languages, the cognitive cost of counting addition up to 20 with doubles (with additional counting up to 3 ) is probably often less than that of using the make-a-ten method. For example, Portuguese textbooks suggest 'doubles', 'doubles plus 1', 'compensation' (e.g. 
$6+8=7+7=14)$ and 'reference numbers' $(6+7=5+1+5+2=10+3=$ 13) in addition calculation and 'counting back', 'the use of tables for the addition to subtraction' and 'identifying inverse operation of subtraction as addition' in the subtraction calculation (Sun et al. 2013). In contrast to the Chinese curriculum, the make-a-ten method, a core concept of addition and subtraction within 20, is often regarded as the foundation of the place value of tens (Sun 2015). It lays the foundations for multi-digit calculation by maintaining coherence between two-digit and multiple-digit addition/subtraction, which may not be the case in most Western curricula because the tens in a teen number name do not play the role of the tens place but are a quantity value role (e.g. $10+3$ is said as thirteen). Most European languages break away from clear regularity in respect to the base-ten place value system, especially with the numbers from 11 to 19 and 19 to 99 (Ejersbo and Misfeldt 2015). Fuson et al. (1997) found that English-speaking children perform badly with the make-a-ten method but suggested that material showing tens in teen numbers might be fruitful.

The Chinese curriculum uses the make-ten method in the first grade to develop the concept of place value and generally assigns at least $30 \mathrm{hr}(1 / 2$ time of the first term) for the conceptual foundation of addition/subtraction, with regrouping as its core practice. Specifically, the make-ten method has been designed critically for the composition of a tens unit, which is also an important aspect of the concept of place value (Ma 1999) and in understanding the concept of addition with regrouping inherited from the principle of the bead calculation tradition (Sun 2015). Ruthven (1998), like many Western scholars, argued that close scrutiny of the mental calculation strategies used by children for the four basic operations suggests that there is no evidence of what is normally understood by place value in their methods. Thompson (1999) claims that mental calculation strategies utilise what has been described as the quantity value aspect of place value (56 seen as 50 and 6), whereas standard written calculations necessitate an understanding of the column value aspect (56 seen as 5 tens and 6 ones). This subtle but important difference has implications for teaching, as it could provide a possible reason why Chinese teachers demonstrate greater conceptual understanding of subtraction in regrouping when decomposing a higher value unit compared with their American counterparts (Ma 1999).

\subsubsection{How to Bridge the Incompatibility: Some Interventions}

Because of the numerous difficulties in number names in many languages, interventions for bridging the incompatibilities between spoken numbers, written numbers and numeration unit numbers are critical. Numeration unit number language in many Eastern countries uses place notion, which is fundamental to the computation. What kinds of didactical language inventions can we use for WNA to better support the conceptual development of numbers and computations?

Several kinds of intervention on this issue were reported at the Macao conference: 
1. Ejersbo and Misfeldt (2015) introduced a didactical linguistic invention: regular number names. These more 'logical' number names are formed with the names of the powers of ten, which are those of units in Danish, like in Chinese. This enhanced the acknowledgement of number structure/regularity and improved the rote learning of number names and computation. A similar invention (Fuson et al. 1997) 'allowed all children to enter the conversation about place-value meanings and 2-digit addition with regrouping before some of them had fully mastered the English number word sequence to 100' (Fuson 2009, p. 346).

2. Sun (2015) introduced a Chinese approach to enhance reasoning by connecting the three core concepts of addition, subtraction and numbers together in the Chinese curriculum. This approach is used in all chapters of addition and subtraction. a. Adding one to a number obtains its adjacent number. b. Subtracting one from the adjacent number gives the original number. By this approach, not only are the three concepts of addition, subtraction and number tied closely together, but connections are formed between them, and the concepts of inverse and equation are developed. This promotes not only doing and memorising but also reasoning. In contrast, in some Western curricula, the ideas of numbers, addition and subtraction are presented in three separate chapters, isolated from one another. This might influence mathematics learning attitudes at the beginning.

3. Considering the French curriculum, Houdement and Tempier (2015) proposed a complete system with a focus on numeration units with which all numbers whole and decimal fractions - can be linked. Among other tasks, they introduced various ways of quantity counting, including one by one and ten by ten in tens.

4. Awareness of the teacher education curricula situation is poor. Azrou (2015) presented the initial step of an intervention project to use difficulties as educational resources. For example, by comparing the French wording of numbers between 11 and 16 with their Arabic or Berber counterparts, students may better realise the anomalous nature of French spoken numbers. By analysing their structure between 60 and 100 in base 20, they may engage in stimulating conversion exercises. From a teaching perspective, developing a clear understanding of these patterns of learning numbers and acknowledging their different characteristics would facilitate effective and meaningful communication between teachers and students and might thus help teachers deal with diversity in the classroom and more effectively scaffold children's learning.

Finally, Sayers and Andrews (2015) developed a simple, eight-dimensional framework through a systematic review of the literature on number sense. Teachers in the Anglophone world often refer to the importance of developing a strong number sense of WNA in elementary school to prepare learners for the adult world (McIntosh et al. 1992). However, psychologists consider number sense innate in all humans (Ivrendi 2011), indicating a gap between what a human is born with and what needs to be taught in elementary school. Sayers and Andrews (2015) developed and tested a new theoretical framework that bridges this spectrum, which they call founda- 
tional number sense, identifying key whole number concepts that require instruction during the first year of schooling.

In short, the foundational ideas that stem from languages and cultures indicate that local number naming can be used to build cultural identities. However, it also causes conflicts between local languages and universal Hindu-Arabic numerals, leading to learning difficulty. Therefore, curriculum and instruction must be bridged to link local languages to universal Hindu-Arabic numerals and calculations. The bridges between the incompatibilities of spoken numbers, written numbers and numeration unit numbers are critical to solutions. In particular, linking numeration unit numbers with spoken numbers, written numbers and the make-a-ten method within 100 may be fundamental for such interventions.

\subsection{Foundational Ideas Influenced by Multiple Communities}

In the previous sections, we provided insights into WNA from both historical and language perspectives. We highlighted how WNA developed from ancient times and how traditional cultural roots can influence and perhaps conflict with the process of learning and teaching mathematics today. According to Morrish (2013), contemporary education must actively seek change for rapid development of the economy, science and technology once they lose their advantage. Attempts at change, often triggered by international comparative studies of mathematics achievement, have had diverse reactions and consequences (Feniger et al. 2012). Such changes by policy makers and educators are interpreted and negotiated through a series of processes framed by a variety of principles and practices (Kanes et al. 2014; Leung 2014; Wiseman 2013).

Mathematics education is embedded in the four major contexts of economics and business, academic mathematics, science and technology and public and private stakeholders, and their influence should not be neglected. In the following, we focus on how the teaching and conceptualisation of WNA have been changed by academic mathematics, science and technology, and the public and stakeholders. The first case concerns the influence of economic and business and ancient China. The other cases concern the influence of academic mathematics, science and technology and public and private stakeholders in modern times in Israel, France and Canada, respectively. These cases seek to understand the how and why of curriculum changes, with a focus on the fundamental losses and gains. The choice of examples is related to reports made by participants at the Macao Conference. 


\subsubsection{The Influence of Economics and Business: A Case from Ancient China}

Sun (2015) discusses how the early Chinese invented number names and calculation tools (counting rods and, later, the suàn pán or Chinese abacus), in which place value is the overarching principle that captures the spirit of WNA (Lam and Ang 2004). This advanced the development of Chinese mathematics. However, rod calculation speed is slow and inconvenient for calculation with large numbers, which is often necessary to calculate labour, capital and products in the markets. To meet the need for efficiency for economics and business development, the Chinese abacus replaced the ancient counting rods, improving calculation speed and efficacy (Sun 2015). The replacement of rods caused a significant deterioration in calculation rationale from the time of rod calculation (Lam and Ang 2004), because the step-by-step procedures using rods were replaced by the 'calculation songs' of bead calculation.

In Tongwen Suanzhi (同文算指 literally meaning 'rules of arithmetic common to cultures'), compiled by the official scholar Li Zhi-zao (李之藻1565-1630) of the Ming court in collaboration with the Italian Jesuit Matteo Ricci (利瑪竇15521610), written calculation that had been in common practice in Europe since the sixteenth century was introduced into the Chinese system. Compared with the methods of traditional bead calculations, the main advantage of written calculation lies in keeping a record of the intermediate steps, enabling easy checking afterwards. It also allows viewing of the procedure, facilitating the understanding of the underlying reasoning without having to memorise what is going on during the calculation. This is difficult to attain in calculation using counting rods or an abacus. Siu (2015) argued that the rationale for learning written calculation, at least once in a person's lifetime, seems to be the acquisition of understanding of the underlying principle of the basic operations in arithmetic, which is essential in future learning. He further warned that using electronic calculators in current primary schools is similar to bead calculation, where the intermediate calculation rationale is hidden. 'Ironically we would be turning back the wheel of history in some sense in that we erase the intermediate steps if we depend on an electronic calculator too much'(p. 137). To meet the growing need for a quick analytical and quantitative approach to problem solving, changes in calculation tools were needed. However, the associated calculation rationale was becoming weaker and thus needed to be addressed.

\subsubsection{The Influence of Academic Mathematics: A Case from the Mathematics Community in Israel}

Considering the depth of their mathematical understanding, it is natural to assume that mathematicians should have a role in the professional development of elementary school teachers. However, mathematicians have little experience of teaching 
WNA. Furthermore, the discourse of university mathematics and its teaching is quite different from its elementary school counterpart. What might happen if members of these communities were to meet and interact? This question was investigated in a professional development (PD) course for in-service elementary school teachers in Israel that was conceived and taught by a professor of mathematics.

Cooper (2015) analysed mathematical and pedagogical discourse as related to a particular mathematical topic - division with remainder (DWR). In school mathematics, DWR is transient: it becomes redundant once students are familiar with the field of rational numbers. In advanced mathematics, DWR is generalised to Euclidian domains. Remainders of DWR are also important as representatives of the ring $\mathrm{Z} / \mathrm{nZ}$. In view of such differences, Cooper asks, how can the meeting of a mathematician and in-service elementary school teachers foster mutual professional growth?

The instructor (Rick) was struck by the problematic aspect of standard notation. The $=$ sign denotes equality, which should be transitive. If we write $25: 3=8(1)$ and $41: 5=8(1)$, transitivity of equality demands that $25: 3=41: 5$, which Rick considered 'complete nonsense'. His proposed solution was to change the notation:

Standard notation in Israel: $25: 3=8(1)$. Proposed notation: $25: 3=8(1: 3)$

The new notation is read 'eight with remainder 1 which needs to be divided by 3 '. To justify the new notation, Rick claimed that $8(1)$ has no meaning as a quantity when the divisor is not known. However, some teachers challenged this, claiming that the 8 and the 1 are 'quantities' and that the equality 25:3=41:5 is an equivalence. Indeed, there is nothing intrinsically wrong with this equivalence relationship, where 8(1) represents a class of DWR exercises that have the same result (quotient and remainder). Thus, the equality is 'complete nonsense' only because it is not consistent with the mathematical horizon of fractions, where $\frac{25}{3}=\frac{41}{5}$ is indeed incorrect. Although they did not agree with Rick regarding the standard notation's deficiencies, the teachers did endorse the new notation for its pedagogical affordance in providing a smooth transition from whole numbers to fractions. Rick emphasised this affordance in presenting the remainder as having the potential to be divided, a potential that can be realised in fair sharing situations in which the unit can be split and will be mathematically realised in fraction arithmetic. Pedagogical affordances were further explored. One teacher attending to procedures at the pedagogical horizon appreciated how the new notation might offer a smooth transition to decimal long division, where students often neglect to divide the remainder. She felt that the new notation, in signalling an unfinished division, would help overcome this difficulty. This episode, one of many described by Cooper (2015), shows how two communities with conflicting perspectives on DWR and its notation jointly explored the mathematical and pedagogical aspects of mathematical notation and worked together to gain a deeper understanding of this surprisingly complex topic, gaining insight that was new to all parties involved. In this way, the community of academic 
mathematics contributed to the professional development of elementary school teachers while at the same time deepening their own understanding of school mathematics and its teaching.

\subsubsection{The Influence of Science and Technology: A Case from the New Math Reform in France}

Science and technology also affect WNA. After the Sputnik crisis, to boost science education, technological development and mathematical skill in the population, New Math was introduced in many countries. Chambris (2015) reported on the New Math reform in France. This international phenomenon (ICMI 2008; Kilpatrick 2012) affected all levels of mathematics teaching in the 1960s and the early 1970s and has had lingering effects. It had two major focuses: (1) teaching 'new' math, including renewing of the mathematical fundamentals of teaching (e.g. Griesel 2007) and (2) taking into account psychological features related to learning and child development. Two famous subjects were introduced in WNA: set theory, an aspect of contemporary math, and numeration bases other than ten (hereafter called bases) (to teach base-ten principles), an aspect of psychology (Kilpatrick 2012; Bruner 1966). This phenomenon may be interpreted in terms of the construct of didactical transposition (Chevallard 1985) - the transformation and adaptation of knowledge produced by the scientific community to make it suitable for use as learning objects. This transformation occurred at the end of the 1970s.

In France, from 1900 to 1960 , the classical theory (history section above) was adapted in close terms in textbooks. Tasks such as 'Write in figures: $3 \mathrm{H} 4$ T 5 O' and 'convert 3 hundreds into tens' - both using the symbolic register of the numeration units - were basic and current. They disappeared as the 'bases' appeared. Students interpreted the positional notation in 'bases' as a procedure, grouping and ungrouping, and they struggled when they had no manipulatives left (ERMEL 1978; Perret 1985). The process is as follows: $\sum r_{i} a^{i}$ becomes $\sum r_{i} 10^{i}$; then, $a \times 1000+b \mathrm{x}$ $100+c \times 10+d$ or $a 000+b 00+c 0+d$. Such 'writings' as $40+7+50+43+25$ also appeared. 'The key issue is to familiarise children with a direct work with the writings' (our translation; ERMEL 1978, p. 17): 1, 10, 100, 1000, etc. played an increasing role. Within a few years, 'write in figures: $3 \mathrm{H} 4 \mathrm{~T} 5 \mathrm{O}$ ' was replaced by 'compute $3 \times 100+4 \times 10+5$ ' or 'compute $300+40+5$ '. Conversions were not replaced. A new symbolic register - that of the powers of ten written in figures - had emerged, and the number 1 became the only unit to be taught.

Within the transposed academic theory, the technique to obtain positional notation from $3 \times 100+4 \times 10+5 \times 1$ is to 'juxtapose $3,4,5$ '. The present implicit rules to obtain it are to (1) multiply by 100 (resp. 10) (write two (resp. one) zeroes on the right) and (2) add numbers (use a 'column algorithm'). That is, put them one under the other, aligned from the right side. In 1995, decompositions with numeration units began to come back. The way to achieve them is $3 \mathrm{H}=300$ (due to the hun- 
dreds place), $4 \mathrm{~T}=40,5 \mathrm{O}=5$; then, compute $300+40+5$, that is, 345 . Thus, in the contemporary period, there is a kind of hybridisation by the units of the transposed academic theory. However, numeration units indicate only the digits' places and their units, and it is common not to find the relation 1 hundred $=10$ tens in present second and third grade textbooks. This provides three interpretations of positional notation: it sums up an additive relation in the latter case, the numbers of units in the classical theory, and serves as a polynomial algebraic relation within the transposed academic theory.

The influence of academic mathematics is apparent within the use of the academic theory. As in the previous case, two kinds of tension between school and academic mathematics arise: (1) different needs (units vs advanced algebra) and (2) different perspectives on a shared sign symbolism (positional notation).

\subsubsection{The Influence of Public and Private Stakeholders: A Case from Current Curriculum Reform in Canada}

Public and private stakeholders also affect WNA. The implementation of international achievement tests such as TIMSS in 1995 and OECD's PISA in 2000 prompted widespread curriculum reform, which often focused extensively on WNA. Multiple stakeholders, including educators, school personnel, business leaders and parents, often expressed conflicting arithmetic goals and pedagogical expectations (Brown and Clarke 2013). These conflicts, called the 'Math Wars', occurred in North America (e.g. Klein 2007), Europe (e.g. Prenzel et al. 2015) and China (Zhao 2005).

Although Canada performs well in international tests, the public and private stakeholders of current curricula have sparked public debate. For example, students are expected to develop flexible and mental mathematics strategies through the use of compensation methods (e.g. $54-37=54-40+3$ ) and properties of numbers and operations (e.g. $8 \times 6=8 \times 3 \times 2$ ) to compute. Through an analysis of online responses to newspaper articles reporting Canada's faltering PISA scores, McGarvey and McFeetors (2015) sought to understand public perspectives. The following quotation is an example of an online comment posted in response to a national news article written by mathematician opposing today's elementary curriculum:

\footnotetext{
I learned things by rote in grade school and then later on in high school I learned how to do abstract problem solving in topics like algebra. From what I understand, the system is unnecessarily complicating kids' minds by saying they shouldn't memorize basic multiplication or learn how to do long division or carry numbers. I don't see the usefulness in that. (McDonald 2013)
}

Keywords in this comment such as 'by rote', 'memorise', 'long division' and 'carry' tend to trigger negative reactions from mathematics educators in much the same way that 'mathematics for understanding', 'strategy-based learning' and 'studentcentred approaches' often trigger negative reactions from the public. 
Rather than dichotomising the arguments, the analysis revealed two sets of shared goals: (1) students need the opportunity to reach expected mathematics goals (e.g. develop computational skills and problem-solving), and (2) essential supports must be in place for students to reach the goals of mathematics learning (e.g. knowledgeable teachers, clearly written teaching resources). Reframing criticism into mutual concerns offers a starting place for conversation that empowers communities to see commonalities in their perspectives of children's mathematics learning. In short, public and private stakeholders in Canada and in other countries have affected WNA by changing policies and goals.

\subsubsection{Foundational Ideas Summary: Understanding the Unpredictable Long-Term Effects of Change}

The four reports above describe the influence of economics and business, academic mathematics, science and technology, the public and stakeholders and the various changes in the conception of WNA and its teaching. For example, in the wake of New Math, the incorporation of academic mathematics as foundational knowledge into school mathematics may lead to fundamental loss of various elements, including numeration units. In contrast, Cooper's example demonstrates how the mathematicians' perspective must undergo didactic transposition to fit the teaching and learning of WNA. Thus, to foster change, school mathematics and academic mathematics should be combined. The professional development described by Cooper can be seen as an important step in the enactment of such a transposition. However, taking the mathematicians' perspective into account in WNA curriculum design is a complex matter. While these examples may not be applicable outside their specific contexts, they may lead us to reflect upon ways in which new goals for WNA might be defined and implemented. However, a number of themes emerge when considering the four cases as a whole related to issues that are likely to arise when defining, implementing and communicating new goals for WNA.

Deep changes seem to occur slowly, perhaps because some features of prior practices persist, but once change has begun, it seems impossible to stop. This is evident in the lingering effects of New Math long after its apparent failure. The arguments used to introduce bases in the New Math curriculum came from psychology. Yet after the turbulent period of reform, during the so-called counter-reform, the "new" mathematics - here represented by an academic theory that is, in fact, not so new - became a mathematical foundation for the teaching of place value. The effects of the changes were probably neither anticipated nor mastered. This also seems to be the case in the changes in China, especially the replacement of rods with beads. When change is implemented, awareness by educators and policy makers is needed because where there are gains, there can also be fundamental losses to elements once taken for granted. This is important because key aspects of a complex 
system may be almost invisible to observers and may be lost when changes are made.

New Math was partly directed by economic issues linked with the growing needs of engineers and scientists in Western societies. PISA studies from the Organisation for Economic Co-operation and Development aim to determine the extent to which 15-year-old students are prepared to face the demands of the society they will live in. Does society need globalised mathematics education and, if so, how will it manifest itself in the future? Are the PISA tests the Tongwen Suanzhi (above) of modern times? Where will the changes develop in relation to the economic needs of society (Siu 2015)? One might suppose that history repeats itself in the redesigning of a WNA curriculum, as it must take into account the evolution of economic societal needs, thus causing tensions such as those described above.

In the examples described above, one or more of the operating communities engaged in a genuine attempt to understand the other's perspective and to promote productive communication. Despite decades of efforts to explain the benefits of current approaches in whole number arithmetic, the arguments have not been convincing for many stakeholders. Seeking ways to identify common goals and to address unfamiliarity with current approaches is an essential step in re-engaging parents and the community in children's learning. A certain failure of New Math reform is acknowledged (Kilpatrick 2012), despite some cooperation between mathematicians, psychologists, math educators and teachers. A lack of teacher education, a lack of resources and incompatibility between academic math and school math are often cited as reasons for this failure. Finally, recurring themes arise as means to achieve changes: bridging communities to share goals and means between stakeholders and the adaptation of teacher education and resources to the new goals.

\subsection{The What and Why of WNA: Towards a Cognitive Dimension}

The historical, cultural and linguistic foundations of WNA set out in this chapter have influenced how an educational system develops its WNA. How students develop fundamental ideas about WNA and what is needed by teachers to nurture such ideas remain themes in the following chapters. However, several papers in this group have examined some of the different representations of how teachers might present WNA at different stages of children's development. The discussions in these papers have provided important insights into the processes that can be evoked in different communities from different perspectives. Other influential aspects, such as humans' innate cognitive abilities, are detailed in Chap. 7, while language, artefacts and tasks used by teachers are instantiated in Chap. 9. The delicate question of teacher education is addressed in Chap. 17. 


\section{References}

Ascher, M., \& Ascher, R. (1981). Ethnomathematics. In A. B. Powell \& M. Frankenstein (Eds.), Ethnomathematics: Challenging Eurocentrism in mathematics (pp. 25-50). Albany: State University of New York Press/SUNY Press.

Askew, M., \& Brown, M. (2001). Teaching and learning primary numeracy: Policy, practice and effectiveness. Nottingham: British Educational Research Association.

Barton, B. (2008). The language of mathematics: Telling mathematical tales. New York/Berlin: Springer.

Baxter, W. T. (1989). Early accounting: The tally and checkerboard. Accounting Historians Journal, 16(2), 43-83.

Bezout, E., \& Reynaud, A.-A.-L. (1821) Traité d'arithmétique à l'usage de la marine et de l'artillerie, 9e édition.

Briand, J., Loubet, M., \& Salin, M.-H. (2004). Apprentissages mathématiques en maternelle. Paris: Hatier.

Brousseau, G. (1997). The theory of didactical situations in mathematics (Didactique des mathematiques, 1970-1990) (N. Balacheff, M. Cooper, R. Sutherland, \& V. Warfield, Eds. and Trans.). Dordrecht: Kluwer Academic Publishers.

Brown, T., \& Clarke, D. (2013). Institutional contexts for research in mathematics education. In M. A. Clements, A. J. Bishop, C. Keitel, J. Kilpatrick, \& F. K. S. Leung (Eds.), Third international handbook of mathematics education (pp. 459-484). New York: Springer.

Bruner, J. S. (1966). Toward a theory of instruction. Cambridge, MA: Harvard University Press.

Cauty, A. (1984). Taxinomie, syntaxe et économie des numérations parlées. Amerindia, 9, 111-146.

Chemla, K. (1996). Positions et changements en mathématiques à partir de textes chinois des dynasties Han à Song-Yuan. Quelques remarques. Extrême-Orient, Extrême-Occident, 18, $115-147$.

Chemla, K. (1998). History of mathematics in China: A factor in world history and a source for new questions. Documenta Mathematica, extra volume ICM 1998, III, 789-798.

Chemla, K. (2007). Observing mathematical practices as a key to mining our sources and conducting conceptual history: Division in ancient China as a case study. In L. Soler et al. (Eds.), Science after the practice turn in the philosophy, history, and social studies of science (Vol. 14, pp. 238-276). London: Routledge.

Chevallard, Y. (1985). La transposition didactique. Grenoble: La Pensée sauvage.

Crump, T. (1990). The anthropology of numbers. Cambridge: University Press.

Dorier, J.-L. (2000). Use of history in a research work on the teaching of linear algebra. In V. Katz (Ed.), Using history to teach mathematics - An international perspective, MAA notes (Vol. 51, pp. 99-110). Washington, DC: The Mathematical Association of America.

El Bouazzaoui, H. (1982). Étude de situations scolaires des premiers enseignements du nombre et de la numération. Relations entre divers caractères de ses situations et le sens, la compréhension de l'apprentissage de ces notions. Doctoral dissertation. Bordeaux: Université de Bordeaux 1.

ERMEL. (1978). Apprentissages mathématiques à l'école élémentaire. Cycle élémentaire. Tome 2. Paris: SERMAP-Hatier.

Feniger, Y., Livneh, I., \& Yogev, A. (2012). Globalisation and the politics of international tests: The case of Israel. Comparative Education, 48(3), 323-335.

Fernandes, L. (2015, January 11). The Abacus: A brief history [Blog post]. Retrieved from http:// www.ee.ryerson.ca/ elf/abacus/history.html

Fuson, K. C. (2009). Avoiding misinterpretations of Piaget and Vygotsky: Mathematical teaching without learning, learning without teaching, or helpful learning-path teaching? Cognitive Development, 24(4), 343-361.

Fuson, K. C., Smith, S. T., \& Lo Cicero, A. (1997). Supporting Latino first graders' ten-structured thinking in urban classrooms. Journal for Research in Mathematics Education, 28(6), 738-766. 
Goswami, U. (2008). Cognitive development. The learning brain. Hove/New York: Psychology Press.

Griesel, H. (2007). Reform of the construction of the number system with reference to Gottlob Frege. ZDM: Mathematics Education, 39(1-2), 31-38.

Groza, V. S. (1968). A survey of mathematics: Elementary concepts and their historical development. New York: Holt, Rinehart and Winston.

Guitel, G. (1975). Histoire comparée des numérations écrites. Paris: Flammarion.

Guo, S. (2010). Chinese history of science and technology. Beijing: Science Press. [In Chinese].

Høyrup, J. (2002). A note on old Babylonian computational techniques. Historia Mathematica, 29(2), 193-198.

ICMI. (2008). The first century of the International Commission on Mathematical Instruction. History of ICMI by Furinghetti, F., \& Giacardi, L. http://www.icmihistory.unito.it

Ifrah, G. (2000). The universal history of numbers: From prehistory to the invention of the computer. New York: Wiley.

Ivrendi, A. (2011). Influence of self-regulation on the development of children's number sense. Early Childhood Education Journal, 39(4), 239-247.

Jankvist, U. T. (2009). A categorization of the "whys" and "hows" of using history in mathematics education. Educational Studies in Mathematics, 71(3), 235-261.

Kanes, C., Morgan, C., \& Tsatsaroni, A. (2014). The PISA mathematics regime: Knowledge structures and practices of the self. Educational Studies in Mathematics, 87(2), 145-165.

Keller, O. (2016). l'invention du nombre. Paris: Garnier.

Kilpatrick, J. (2012). The new math as an international phenomenon. ZDM: Mathematics Education, 44(4), 563-571.

Klein, D. (2007). A quarter century of US "math wars" and political partisanship. BSHM Bulletin, $22,22-33$.

Lam, L. Y., \& Ang, T. S. (2004). Fleeting footsteps: Tracing the conception of arithmetic and algebra in ancient China. Singapore: World Scientific.

Leung, F. K. (2014). What can and should we learn from international studies of mathematics achievement? Mathematics Education Research Journal, 26(3), 579-605.

Leung, F. K., Graf, K. D., \& Lopez-Real, F. J. (Eds.). (2006). Mathematics education in different cultural traditions: A comparative study of East Asia and the West: The 13th ICMI study. New York: Springer.

Ma, L. (1999). Knowing and teaching elementary mathematics: Teachers' understanding of fundamental mathematics in China and the United States. Mahwah: Lawrence Erlbaum Associates.

Mainzer, K. (1991). Natural numbers, integers and rational numbers. In H.-D. Ebbinghaus et al., Numbers (with an introduction by K. Lamotke; H.L.S. Orde, Trans.; J.H. Ewing, Ed.) (pp. 9-26). New York: Springer. (Original work published 1983)

Margolinas, C., \& Wozniak, F. (2012). Le nombre à l'école maternelle: Une approche didactique. Bruxelles: De Boeck.

Martzloff, J.-C. (1997). A history of Chinese mathematics. Berlin-Heidelberg: Springer.

May, K. O. (1973). Bibliography and research manual of the history of mathematics. Toronto: University of Toronto Press.

McDonald, M. (2013, September 13). Frustrated professors convince elementary schools to step back from "new math" and go "back to basics". The National Post. Retrieved from http://news.nationalpost.com/2013/09/13/frustrated-professors-convince-schools-to-stepback-from-new-math-and-go-back-to-basics/

McIntosh, A., Reys, B., \& Reys, R. (1992). A proposed framework for examining basic number sense. For the Learning of Mathematics, 12(3), 2-8.

Menninger, K. (1969). Number words and number symbols: A cultural history of numbers. Cambridge, MA: The MIT Press. (Translated from the German edition of 1958).

Miller, K. F., \& Zhu, J. (1991). The trouble with teens: Accessing the structure of number names. Journal of Memory and Language, 30(1), 48-68.

Morrish, I. (2013). Aspects of educational change (RLE Edu D). London: Routledge.

Needham, J. (1959). Science and civilisation in China. Vol. 3: Mathematics and the sciences of the heavens and the earth. Cambridge: Cambridge University Press. 
Perret, J. F. (1985). Comprendre l'écriture des nombres. Bern: P. Lang.

Prenzel, M., Blum, W., \& Klieme, E. (2015). The impact of PISA on mathematics teaching and learning in Germany. In K. Stacey \& R. Turner (Eds.), Assessing mathematical literacy: The PISA experience (pp. 239-248). Cham: Springer International.

Proust, C. (2008). Quantifier et calculer: Usages des nombres à Nippur. Revue d'histoire des mathématiques, 14(2), 143-209.

Proust, C. (2009). Numerical and metrological graphemes: From cuneiform to transliteration. Cuneiform Digital Library Journal, 1.

Ruthven, K. (1998). The use of mental, written and calculator strategies of numerical computation by upper primary pupils within a 'the calculator-aware' number curriculum. British Educational Research Journal, 24(1), 21-42.

Ryan, J. (1827). An elementary treatise on arithmetic, in theory and practice. New York: Collins \& Hannay.

Saxe, G. B. (1981). Body parts as numerals: A developmental analysis of numeration among the Oksapmin in Papua New Guinea. Child Development, 52(1), 306-316.

Schmandt-Besserat, D. (1992). Before writing (Vol. 2). Austin: University of Texas Press.

Seidenberg, A. (1962). The ritual origin of counting. Archive for History of Exact Sciences, 2(1), $1-40$.

Sun, X., Neto, T. B., \& Ordóñez, L. E. (2013). Different features of task design associated with goals and pedagogies in Chinese and Portuguese textbooks: The case of addition and subtraction. In C. Margolinas (Ed.), Task design in mathematics education. Proceedings of ICMI study 22, July 2013, Oxford, United Kingdom (pp. 409-418). Retrieved from https://hal.archivesouvertes.fr/hal-00834054v3

Tempier, F. (2013). La numération décimale de position à l'école primaire: une ingénierie didactique pour le développement d'une ressource. Doctoral dissertation. Paris: Université de Paris Diderot - Paris 7.

Thompson, I. (1999). Implications of research on mental calculation for the teaching of place value. Curriculum, 20(3), 185-191.

Wiseman, A. W. (2013). Policy responses to PISA in comparative perspective. In H.-D. Meyer \& A. Benavot, PISA, power and policy. The emergence of global educational governance (pp. 303-322). Oxford: Symposium Books.

Zhao, Y. (2005). Increasing math and science achievement: The best and worst of the east and west. Phi Delta Kappan, 87(3), 219-222.

\section{Cited papers from Sun, X., Kaur, B., \& Novotna, J. (Eds.). (2015). Conference proceedings of the ICMI study 23: Primary mathematics study on whole numbers. Retrieved February 10, 2016, from www.umac.mo/fed/ICMI23/doc/Proceedings_ICMI_ STUDY_23_final.pdf}

Azrou, N. (2015). Spoken and written arithmetic in post-colonial country: The case of Algeria (pp. 44-51).

Bass, H. (2015). Quantities, numbers, number name, and number line (pp. 10-20).

Beckmann, S., Izsák, A., \& Ölmert, I. B. (2015). From multiplication to proportional relationships (pp. 518-525).

Cao, Y. Li, X., \& Zuo, H. (2015). Characteristics of multiplication teaching of whole numbers in China: The application of the nine times table (pp. 423-430).

Chambris, C. (2015). Mathematical foundations for place value throughout one century of teaching in France (pp. 52-59).

Changsri, N. (2015). First grade students' mathematical ideas of addition in the context of lesson study and open approach (pp. 60-67). 
Cooper, J. (2015). Combining mathematical and educational perspectives in professional development (pp. 68-75).

Dorier, J.-L. (2015). Key issues for teaching numbers within Brousseau's theory of didactical situations (pp. 76-83).

Ejersbo, L., \& Misfeldt, M. (2015). The relationship between number names and number concepts (pp. 84-91).

González, S., \& Caraballo, J. (2015). Native American cultures tradition to whole number arithmetic (pp. 92-98).

Hodgson, B. R., \& Lajoie, C. (2015). The preparation of teachers in arithmetic: A mathematical and didactical approach (pp. 307-314).

Houdement, C., \& Tempier, F. (2015). Teaching numeration units: Why, how and limits (pp. 99-106).

McGarvey, L., \& McFeetors, J. (2015). Reframing perceptions of arithmetic learning: A Canadian perspective (pp. 115-123).

Sayers, J., \& Andrews, P. (2015). Foundational number sense: The basis of whole number arithmetic competence (pp. 124-131).

Siu, M. K. (2015). Pedagogical lessons from Tongwen Suanzhi (同文算指) - transmission of Busan (算 written calculation) in China (pp. 132-139).

Sun, X. (2015). Chinese core tradition to whole number arithmetic (pp. 140-148).

Thanheiser, E. (2015). Leveraging historical number systems to build an understanding of base 10 (pp. 149-156).

Zou, D. (2015). Whole number in ancient Chinese civilisation: A survey based on the system of counting-units and the expressions (pp. 157-164).

Open Access This chapter is licensed under the terms of the Creative Commons Attribution 4.0 International License (http://creativecommons.org/licenses/by/4.0/), which permits use, sharing, adaptation, distribution and reproduction in any medium or format, as long as you give appropriate credit to the original author(s) and the source, provide a link to the Creative Commons license and indicate if changes were made.

The images or other third party material in this chapter are included in the chapter's Creative Commons license, unless indicated otherwise in a credit line to the material. If material is not included in the chapter's Creative Commons license and your intended use is not permitted by statutory regulation or exceeds the permitted use, you will need to obtain permission directly from the copyright holder.

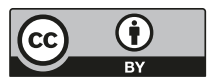

